$A \bar{n} \overline{\mathrm{A}} \overline{\mathrm{d}} \overline{\mathrm{C}} \overline{\mathrm{C}}|\mathrm{A}| \begin{aligned} & \text { Appareil } \\ & \mathbf{1 6} \mid \mathbf{2 0 1 5} \\ & \text { Individuer Simondon. De la redécouverte aux } \\ & \text { prolongements }\end{aligned}$

\title{
La contribution de Gilbert Simondon au naturalisme
}

Giovanni Carrozzini

\section{OpenEdition}

Journals

Édition électronique

URL : http://journals.openedition.org/appareil/2206

DOI : 10.4000/appareil.2206

ISSN : 2101-0714

Éditeur

MSH Paris Nord

Référence électronique

Giovanni Carrozzini, «La contribution de Gilbert Simondon au naturalisme », Appareil [En ligne], 16 |

2015, mis en ligne le 09 février 2016, consulté le 30 juillet 2020. URL : http://journals.openedition.org/ appareil/2206 ; DOI : https://doi.org/10.4000/appareil.2206

Ce document a été généré automatiquement le 30 juillet 2020.

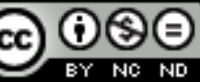

Appareil est mis à disposition selon les termes de la Licence Creative Commons Attribution - Pas d'Utilisation Commerciale - Pas de Modification 4.0 International. 


\title{
La contribution de Gilbert Simondon au naturalisme
}

\author{
Giovanni Carrozzini
}

\section{Introduction : sur le naturalisme comme antidote à l'anthropocentrisme et à l'anthropologie philosophique}

1 La plupart des notions employées par Simondon ont été élaborées à partir de contributions théoriques issues des sciences qui lui étaient contemporaines. Par ailleurs, la philosophie de Simondon manifeste une disposition de fond expressément non anthropocentrique, et qui s'oppose même à toute anthropologie philosophique. Le premier de ces aspects permet, à mon sens, d'inscrire la réflexion de Simondon dans la sphère théorique du naturalisme; le second d'assimiler cette réflexion au naturalisme ontologique subjectif tel que Huw Price l'a défini.

2 Par «naturalisme » on entend l'horizon philosophique qui vise à « associer strictement la philosophie avec la science ${ }^{1} »$. Toutefois, ce terme ne possède pas une signification précise et univoque dans la philosophie contemporaine. David Papinau, rédacteur de l'entrée « Naturalism » dans la Standford Encyclopedia of Philosophy, précise que le sens actuel de ce concept découle des débats qui se sont déroulés aux États-Unis à partir de la première moitié $\mathrm{du} \mathrm{xx}^{\mathrm{e}}$ siècle, et qu'il faut distinguer entre une composante ontologique et une composante méthodologique. Le naturalisme ontologique "affirme que dans la réalité il n'y a pas de place pour des entités "surnaturelles" ou "fantasmagoriques", tandis que le naturalisme méthodologique "reconnaît une espèce d'autorité générale à la méthode scientifique ${ }^{2 "}$ " .

3 Dans le cadre du naturalisme ontologique, il semble particulièrement intéressant de considérer la position de Huw Price, et d'emprunter sa définition du «naturalisme subjectif » dans sa distinction d'avec le «naturalisme objectif». Ce dernier peut être défini comme une perspective philosophique visant à réconcilier le monde du langage ordinaire et celui décrit par la science, grâce à une approche "représentationaliste " selon laquelle les énoncés « représentent » ou « se trouvent à la place » des aspects du 
monde. Le naturalisme subjectif, au contraire, serait anti-représentationaliste et "quasi-réaliste ${ }^{3}$ » : c'est un "point de vue philosophique développé à partir de la compréhension selon laquelle nous, les humains, faisons partie sans aucun doute du monde naturel ${ }^{4} »$. En conséquence :

$\left[l^{\prime}\right]$ un des succès majeurs obtenus par la science est d'avoir individué de nouvelles modalités pour nous démontrer combien nous sommes insignifiants, du point de vue du monde - combien est idiosyncrasique l'angle sous lequel nous cherchons à lui assigner un sens. Nous, les humains, pourrions trouver inquiétant cet enseignement, mais la science semble avoir prospéré grâce à ce régime d'humilité auto-imposés.

Le naturalisme ontologique subjectif de Price est donc un " enseignement d'humilité " pour le "genre humain », car il exclut tout prétendu primat de ce dernier sur d'autres manifestations de la nature. Les hommes y sont conçus comme des manifestations éphémères de cette nature qui constitue l'objet privilégié des sciences. Autrement dit, pour se dire "naturaliste" du point de vue ontologique subjectif, il ne suffit pas de reconnaître la valeur des résultats obtenus par les sciences naturelles (en étendant leur méthode de recherche aux disciplines qui s'occupent spécifiquement de l'être humain) et de vouloir accorder pensée philosophique et pensée scientifique; il faut aussi se défaire de toute prétention anthropocentrique et anthropologique au sens de l'anthropologie philosophique.

Carlo Rovelli, physicien italien qui travaille à Marseille dans le cadre de la théorie de la gravitation quantique à boucles (« loop quantum gravity») - l'une des théories visant à fonder la gravité quantique -, a récemment écrit à propos du naturalisme :

[pour] un physicien théorique comme moi, habitué à penser l'étendue sans bornes de plus de cent milliards de galaxies, chacune formée de plus de cent milliards d'étoiles, chacune avec sa guirlande de planètes, sur l'une desquelles nous ne sommes qu'un phénomène bref et fugace, grains de poussière perdus dans le cosmos sans bornes, tout cela est tout à fait trivial. Tout type d'anthropocentrisme pâlit face à cette immensité. Le naturalisme, c'est ça ${ }^{6}$.

6 En ce sens, le naturalisme ontologique peut être conçu comme l'arrière-plan théorique de la philosophie de la nature qui s'exprime dans Lindividuation à la lumière des notions de forme et d'information. La tendance de Simondon à accorder la philosophie avec la science est ce qui situe sa réflexion dans le cadre du naturalisme ontologique. Son antianthropocentrisme et son refus de toute anthropologie philosophique l'apparentent au naturalisme ontologique subjectif - ce dernier n'étant pas seulement une manière de s'opposer aux discours anthropocentriques mais aussi une orientation qui s'oppose à toutes les philosophies qui reconnaissent une essence humaine et séparent l'homme de la nature, lui attribuant une différence spécifique essentielle. Il ne faut pas, cependant, dériver du naturalisme de Simondon une conséquence nihiliste : le fait de reconnaître le genre humain comme un mode fini de la nature (pour le dire à la manière de Spinoza) ne constitue pas un acte de négation de l'humanité de l'homme. Chez Simondon, le naturalisme s'émancipe de tout anthropocentrisme et de toute anthropologie philosophique tout en restant un humanisme d'un certain type.

7 Réfléchissant aux destins possibles de la civilisation contemporaine, Rovelli précise pour sa part :

[...] de même que nous sommes capables de faire face, plus ou moins bien, à notre mort individuelle, de même nous ferons face à l'écroulement de notre civilisation. Ce n'est pas très différent. Et ce ne sera, certes, pas la première civilisation qui s'écroulera. [...] Nous naissons et nous mourons comme naissent et meurent les 
étoiles, à la fois individuellement et collectivement. Telle est notre réalité. Pour nous, en raison de notre nature éphémère, la vie est précieuse. [...] Mais plongés dans cette nature qui nous a fait et qui nous entraîne, nous nous sentons des êtres sans demeure. [...] Non : nous sommes chez nous. [...] Notre connaissance du monde continue à grandir. Il y a des frontières où nous sommes en train d'apprendre, et où brûle notre désir de savoir. Elles sont là, dans les moindres profondeurs du tissu de l'espace, dans les origines du cosmos, dans la nature du temps, dans le destin des trous noirs, et dans le fonctionnement de notre pensée elle-même ${ }^{7}$.

S'il refuse de reconnaître à l'homme un quelconque privilège ontologique par rapport aux autres manifestations de la nature, le naturalisme n'exclut pas l'humanisme, puisqu'il implique une évaluation des efforts humains pour connaître et comprendre. Il vise plutôt à modérer les excès des « discours sur l'Homme "; il est un acte d'honnêteté intellectuelle par rapport au genre humain, basé sur une perspective antianthropocentrique et opposé, en même temps, à toute anthropologie philosophique. C'est en ce sens que Simondon peut revendiquer un "nouvel humanisme », compatible avec le naturalisme ontologique subjectif tel que défini par Price : un humanisme qui ne débouche jamais sur une exaltation euphorique de l'homme et de sa centralité dans la nature. L'humanisme simondonien est un «mixte de maturité et de jeunesse, si l'on définit par maturité le sens de la mesure interne et par jeunesse le sens de l'enthousiasme ${ }^{8} "$; c'est la même mesure et le même enthousiasme que ceux entrevus dans la réflexion de Carlo Rovelli sur la place de l'homme dans la nature. C'est en vertu de cette modération intrinsèque que la philosophie de la nature élaborée par Simondon, bien qu'humaniste, ne tombe jamais dans l'anthropocentrisme. Sa pensée ne se présente pas seulement comme contraire à tout anthropocentrisme, elle se veut aussi en désaccord avec toute anthropologie philosophique ${ }^{9}$.

Or, en soutenant les raisons du naturalisme contre toutes ces approches qui nient la valeur de la pensée scientifique, Rovelli polémique aussi avec Heidegger, symbole des penseurs anti-naturalistes $\mathrm{du} \mathrm{xx}^{\mathrm{e}}$ siècle qui ne parviennent pas à concevoir l'homme comme simple manifestation de la nature, et cherchent par conséquent à le " couper » à tout prix du reste du vivant. Se référant donc expressément à Heidegger, Rovelli en vient aux résistances rencontrées par le naturalisme en Italie :

[...] elle est diffusée dans notre pays, l'opinion strictement anti-naturaliste selon laquelle «il faut qu'il y ait "quelque chose" au-delà de ce qu'on peut étudier de manière scientifique ». Notre école est structurée par l'idéalisme de Croce, nos philosophes adorent Heidegger [...]. Dans ce climat, il ne faut pas s'étonner si nos intellectuels les meilleurs se tiennent à distance du naturalisme ${ }^{10}$.

Si l'on se base sur les reproches que son élève - peut-être son critique le plus sévère Günther Anders lui avait adressés dans ses notes des années 1930, il est tout à fait justifié d'entrevoir la présence d'une anthropologie philosophique masquée chez Heidegger, surtout chez le Heidegger de Sein und Zeit, auquel semble se référer plus spécifiquement Anders ${ }^{11}$. Il est vrai que dans son ouvrage de 1927, le philosophe de Messkirch avait accordé à l'homme un privilège singulier, ce-dernier étant le «berger de l'être ", le seul qui puisse établir un rapport avec l'être. Or, Günther Anders précise aussi que le manque d'intérêt, de la part de Heidegger, pour la philosophie de la nature s'expliquerait précisément à partir de son anthropologie philosophique inavouée :

[notre] philosophie [...] est restée en tout cas pré-copernicienne et elle vient d'un seul grand égocentrisme vaniteux. C'est surtout à la floraison et à la prospérité de l'« anthropologie philosophique ", aujourd'hui tant aimée, que nous avons participé et continuons de participer chaque jour. Naturellement [...] Heidegger aussi y prend 
part, même s'il déclare avec insistance qu'il ne construit aucune " anthropologie philosophique » et qu'il reste en dehors de cette « orientation à la mode » [...]. Sans aucune différence par rapport aux « anthropologues philosophes » qu'il méprise, il ne prétend pas seulement que nous sommes, mais aussi que nous avons une « essence". Ce mot philosophique du dimanche me pose problème ${ }^{12}$.

11 C'est à cause de cette anthropologie philosophique cachée, ajoute Anders, que Heidegger se serait intéressé « exclusivement à l'existence" humaine en un sens tout à fait anthropocentrique, de manière pré-copernicienne, et non pas au monde en lui-même, au sens de la philosophie de la nature, ce monde qui est toujours le cadre supérieur dans lequel se déroule l'existence humaine ${ }^{13}{ }^{\prime \prime}$.

Il est donc possible de révéler chez Heidegger à la fois une veine résiduelle d'anthropologie philosophique et un certain anthropocentrisme de fond ${ }^{14}$. Il existe une distance infranchissable entre une philosophie telle que celle de Heidegger, qui rejette toute forme de philosophie de la Nature, et celle de Simondon. Le naturalisme ontologique subjectif, conçu comme perspective philosophique niant la spécificité de l'essence humaine et la coupure entre l'être humain et les autres espèces, ne peut que s'opposer aux prémisses théoriques qui, même de manière cachée ou masquée, se trouvent au cœur même de la pensée de Heidegger. Simondon n'accorde à l'homme aucune différence spécifique tranchée par rapport aux autres êtres vivants, aucune essence : l'homme est seulement doté d'un degré de complexité majeur.

13 Le naturalisme en général, comme nous l'avons vu, est un effort théorique qui vise à réconcilier la pensée philosophique avec la pensée scientifique, et c'est justement dans cette perspective qu'il faut placer la recherche simondonienne, celle-ci s'alimentant en effet aux contributions des sciences de son temps pour penser l'ontogenèse comme " passage de la virtualité à l'actualité ${ }^{15}$ ». C'est en cela que Simondon me semble bien saisir la suggestion de Gaston Bachelard affirmant que " [la] culture scientifique doit déterminer des modifications profondes de la pensée ${ }^{16}$ ". La notion simondonienne d' individuation surgit en effet dans un contexte marqué en profondeur par l'exigence de concilier les recherches réalisées dans le domaine de la pensée réflexive avec celles des sciences naturelles et appliquées, qui inspire à Simondon la nécessité de repenser l'individualité et sa place dans la nature. L'individuation, chez Simondon, «n'est pas la rencontre d'une forme et d'une matière préalables existant comme termes séparés antérieurement constitués, mais une résolution surgissant au sein d'un système métastable riche en potentiels : forme, matière et énergie préexistent dans le système ${ }^{17}$ ". Les notions d'équilibre métastable et d'énergie potentielle sont tributaires des découvertes de la physique contemporaine. Comme le note Simondon :

[1]'individuation n'a pu être adéquatement pensée et décrite parce qu'on ne connaissait qu'une seule forme d'équilibre, l'équilibre stable; on ne connaissait pas l'équilibre métastable [...]. Les Anciens ne connaissaient que l'instabilité et la stabilité, le mouvement et le repos, ils ne connaissaient pas nettement et objectivement la métastabilité. Pour définir la métastabilité, il faut faire intervenir la notion d'énergie potentielle d'un système, la notion d'ordre, et celle d'augmentation de l'entropie ${ }^{18}$.

14 Après l'individuation physique et l'individuation vitale simple, l'être humain, à son tour, est en tant qu'individu le produit d'une individuation qui s'est opérée dans le domaine du vivant et qui ne présente, par rapport à l'individuation des autres êtres vivants, que des différences d'échelle, de degré :

[...] il est probable que les animaux se trouvent parfois en situation psychique.

Seulement, ces situations qui conduisent à des actes de pensée sont moins 
fréquentes chez les animaux. L'homme, disposant de possibilités psychiques plus étendues, en particulier grâce aux ressources du symbolisme, fait plus souvent appel au psychisme; c'est la situation purement vitale qui est chez lui exceptionnelle, et pour laquelle il se sent plus démuni. Mais il n'y a pas là une nature, une essence permettant de fonder une anthropologie; simplement, un seuil est franchi : l'animal est mieux équipé pour vivre que pour penser, et l'homme pour penser que pour vivre. Mais l'un et l'autre vivent et pensent, de façon courante ou exceptionnelle ${ }^{19}$.

\section{Sur la notion de relation constituante chez Simondon}

C'est surtout par sa pensée de la relation que l'ontologie simondonienne se distingue. C'est en effet en raison de cette notion de relation que le naturalisme simondonien ne se traduit pas seulement en une perspective qui vise à exploiter les succès obtenus par les sciences de son temps, mais constitue aussi une véritable "réserve de potentiels" pour instaurer des liens féconds avec les hypothèses scientifiques actuelles, telles que celles formulées dans le domaine de la physique en vue de la construction de la théorie de la « gravité quantique unifiée ${ }^{20}$ ».

Avant d'examiner les rapports qu'il est possible d'établir entre la réflexion de Simondon et la théorie de la gravitation quantique, rappelons la définition de la relation constituante selon Simondon : « la relation ne jaillit pas entre deux termes qui seraient déjà des individus; elle est un aspect de la résonance interne d'un système d'individuation; elle fait partie d'un état de système ${ }^{21} »$. Autrement dit, la relation ne s'établit pas entre deux individus préexistants, mais, ayant valeur d'être, elle joue un rôle dans l'individuation elle-même :

La relation, pour l'individu, a valeur d'être ; on ne peut distinguer l'extrinsèque de l'intrinsèque ; ce qui est vraiment et essentiellement l'individu est la relation active, l'échange entre l'extrinsèque et l'intrinsèque; il $\mathrm{y}$ a extrinsèque et intrinsèque par rapport à ce qui est premier. Ce qui est premier est ce système de résonance interne, singulière, de la relation allagmatique entre deux ordres de grandeur ${ }^{22}$.

Le système de résonance interne fonde le processus de constitution de l'individu qui, avec son milieu associé, est le résultat de cette individuation. L'individu se définit donc comme la « réalité d'une relation constituante »:

[la] relation est une modalité de l'être ; elle est simultanée par rapport aux termes dont elle assure l'existence. Une relation doit être saisie comme relation dans l'être, relation de l'être, manière d'être et non simple rapport entre deux termes que l'on pourrait adéquatement connaître au moyen de concepts parce qu'ils auraient une existence effectivement séparée ${ }^{23}$.

18 La relation est l'«impensé", la zone obscure du substantialisme et même de l'hylémorphisme, qui s'était efforcé de penser l'ontogenèse. La pensée relationnelle combat la nécessité de poser tout principium individuationis avant le processus de structuration d'un individu. Elle amorce une véritable révolution conceptuelle dans l'analyse de l'ontogenèse, en nous obligeant à nous situer à l'intérieur du processus génétique et non plus au dehors ${ }^{24}$. Le «réalisme des relations ${ }^{25}$ » simondonien prolonge l'anti-substantialisme bachelardien et se nourrit d'une réflexion sur la physique quantique ${ }^{26}$. Simondon écrit à cet égard que la "relation constituante» est un type de relation que «ni Platon ni Aristote ne connaissaient, et dont le modèle est fourni [...] par la Physique du début du $\mathrm{xx}^{\mathrm{e}}$ siècle $^{27}$ ». Simondon apprécie en particulier la contribution de la "théorie de la 
double solution » de Louis de Broglie. En examinant plus généralement la théorie des quanta et son originelle Weltanschauung, Simondon affirme qu' « un échange d'énergie se fait par quantités élémentaires, comme s'il y avait une individuation de l'énergie dans la relation entre particules, que l'on peut en un sens considérer comme des individus physiques $^{28}$ ". Mais cette notion même d'individu change de manière tout à fait radicale, grâce au rôle joué par la « relation constituante ». Par « individu », il ne faut plus désigner une entité insécable, un " terme constitué », mais la "réalité d'une relation constituante, non [l'] intériorité d'un terme constitué29 ».

La valeur d'être de la relation fait donc de l'individu la manifestation concrète d'une relation, la structure d'une relation ayant valeur génétique. L'individu, de ce point de vue, perd tout caractère d'absolu : sa relativité tient au fait qu'il s'entend ici comme le produit partiel d'une opération d'individuation dont il résulte, comme en résulte également son milieu associé lui-même. Ce milieu est en effet «le complément de l'individu par rapport au tout originel. L'individu seul n'est donc pas le type même de l'être; il ne peut pour cette raison soutenir de relation en tant que terme avec un autre terme symétrique $e^{30} »$.

\section{La relation a valeur d'être : Simondon et la théorie de lagravité quantique}

21 En quoi ces réflexions sur le rôle constitutif de la relation sont-elles, ainsi que nous l'annoncions, prolongées par les hypothèses récemment formulées dans le domaine physique de la gravité quantique? La théorie de la gravitation quantique résulte de l'exigence de concilier les résultats de la théorie de la relativité générale d'Einstein avec les résultats de la physique quantique. Différentes perspectives s'affrontent autour de cet enjeu d'une réunification, en particulier la théorie des cordes (ou des supercordes) et la théorie de la gravité quantique à boucles, ou loop. Le but est d'arriver à une "théorie du Tout ", comme on la désigne dans le monde anglo-saxon. Simondon, dont la préférence allait manifestement à la mécanique ondulatoire développée par Broglie, écrivait quant à lui :

[que] la conception d'Einstein sur les particules et celles de Louis de Broglie exposées dans la théorie de la double solution [pourraient être unies] : ainsi serait réalisée une «synthèse grandiose » de la Relativité et des Quanta ${ }^{31}$.

La conciliation des théories quantique et relativiste permettrait de jeter ensuite les bases d'une vision unifiée de la nature, où s'alimenteraient les recherches conduites dans d'autres domaines scientifiques.

La théorie de la gravité quantique ne se développe pas dans la continuité de la «théorie de la double solution » défendue par de Broglie. En se concentrant spécifiquement sur la nature de l'espace qui, en dernière analyse, constitue l'un des aspects les plus problématiques pour l'unification des perspectives einsteinienne et quantique, Carlo Rovelli résume ainsi l'inspiration de cet effort conduit dans le domaine de la physique :

[...] si l'on combine les idées de base de la relativité générale et de la mécanique quantique, il s'ensuit immédiatement que l'espace, en tant que champ (le champ gravitationnel découvert par Einstein), doit avoir lui aussi une structure granulaire, à l'instar du champ électromagnétique. Il doit y avoir des "grains d'espace ». De plus, la dynamique de ces grains d'espace doit être probabilitaire ${ }^{32}$. 
Quel est le lien entre la notion simondonienne de relation constituante et cette hypothèse théorique qui vise à la « synthèse grandiose » de la physique einsteinienne et de la physique quantique ? Rovelli l'explique lui-même, nous conduisant en plusieurs étapes à cette étonnante référence à l'apeiron d'Anaximandre, qu'il partage avec Simondon. D'abord, énumérant les découvertes faites dès la naissance de la théorie des quanta, avec Planck, Bohr, de Broglie, Schrödinger et Dirac, le physicien s'arrête sur le caractère relationnel de la réalité qu'ils ont théorisée :

[...] la théorie ne décrit pas les choses telles quelles «sont » : elle décrit la manière dont les choses «se passent » et dont elles s'influencent les unes les autres. [...] La réalité est réduite à l'interaction. [...] La mécanique quantique nous enseigne à ne pas penser le monde en termes de «choses » qui se trouvent dans tel ou tel état, mais en termes de " processus $\|^{33}$.

Selon Rovelli, le désaccord d'Einstein avec les derniers résultats de la théorie des quanta dérivait précisément du fait que malgré le caractère relationnel de la théorie de la relativité, il n'arrivait pas à reconnaître jusqu'au bout la nature relationnelle de la réalité; "Einstein ne voulait pas céder sur un point qu'il pensait être décisif: l'existence d'une réalité objective indépendante des interactions ${ }^{34} »$. En ce sens, la contribution simondonienne aurait pour mérite de nous faire comprendre que reconnaître une valeur d'être à la relation, cela signifie, en fait, transformer de manière tout à fait radicale la signification de cette relation, par-delà l'apport d'Einstein luimême.

La "synthèse grandiose " semblerait réalisable, selon Rovelli et les physiciens de la théorie de la gravitation quantique à boucles, en assumant, donc, la nature granulaire de l'espace lui-même, à la condition de ne pas assigner d'autre part à ces grains d'espace le caractère d'« individus constitués »:

[...] l'espace physique est le tissu qui résulte du pulluler continu de cette trame de relations. En soi, les lignes [de champ] ne sont nulle part, elles ne sont dans aucun lieu : ce sont elles, dans leurs interactions, qui créent les lieux. L'espace est créé par l'interaction de quanta de gravité35.

Cela implique évidemment une nouvelle "vision de la nature »: "[le] monde, les particules, l'énergie, l'espace et le temps, tout cela n'est que la manifestation d'un seul type d'entité: les champs quantiques covariants». Les quanta ne sont que des manifestations probables des champs dont ils sont la structure, et la réalité tout entière peut être pensée en termes de «champs quantiques covariants [qui] représentent la meilleure description que nous ayons de l'apeiron, la substance primordiale qui forme le tout, théorisée par le premier savant et philosophe : Anaximandre ${ }^{36} »$.

Manquent encore des confirmations expérimentales pour pouvoir élaborer une loi à partir de ces hypothèses et $\mathrm{du}$ formalisme dans lequel elles s'expriment. Le naturalisme, d'ailleurs, précisément en raison de son affinité avec la pensée scientifique, est un acte d'humilité par rapport à chaque prétention conclusive de la connaissance :

Le travail scientifique que j'ai fait jusqu'ici pourrait ne mener à rien : il pourrait se révéler erroné. Ou bien il pourrait, comme je l'espère, être un petit pas vers la compréhension du monde. Dans un cas comme dans l'autre, les heures indéfinies passées devant les tableaux noirs pleins de formules, ou les discussions avec d'innombrables amis, collègues et étudiants de tous pays du monde, ont été magnifiques, et je suis heureux de les avoir vécues ${ }^{37}$. 
La science, le naturalisme et l'« humanisme nouveau » prôné par Simondon convergent en nous offrant un portrait dynamique de la connaissance, dont le chemin implique un élargissement des recherches sur la nature. L'inachèvement de l'homme qui s'y révèle est en définitive « source de pensée et instrument d'action ${ }^{38}$ ».

\section{BIBLIOGRAPHIE}

Anders Günther, Die Kirschenschlacht. Dialoge mit Hannah Arendt, München, Verlag C.H. Beck oHG, 2012.

Bachelard Gaston, La philosophie du non. Essai d'une philosophie du nouvel esprit scientifique, Paris, Puf., 1940.

Barthélémy Jean-Hugues, Penser l'individuation. Simondon et la philosophie de la nature, Paris, L'Harmattan, 2005.

Barthélémy Jean-Hugues, « La question de la non-anthropologie », in Jean-Marie Vaysse (éd.), Technique, monde, individuation. Heidegger, Simondon, Deleuze, Hidesheim, Georg Olms Verlag, 2006, p. 117-132.

Barthélémy Jean-Hugues, Simondon ou l'Encyclopédisme génétique, Paris, Puf, 2008.

Barthélémy Jean-Hugues, Simondon, Paris, Les Belles Lettres, 2014.

Bontems Vincent, Bachelard, Paris, Les Belles Lettres, 2012.

Carrozzini Giovanni, Gilbert Simondon: Per un'assiomatica dei saperi. Dall'ontologia dell'individuo" alla filosofia della tecnologia, San Cesario di Lecce Manni Editori, 2006.

Carrozzini Giovanni, « Technique et humanisme. Günther Anders et Gilbert Simondon », Appareil, $\mathrm{n}^{\circ}$ 2, 2008, http://revues.mshparisnord.org/appareil/index.php?id=576.

Carrozzini Giovanni, "Essere è relazione. Il realismo di Gilbert Simondon”, dans Carlo Vinti, Fabio Minazzi, Matteo Negro, Agostino Carrino (a cura di), Le forme della razionalità tra realismo $e$ normatività, Milan, Mimesis, 2009, p. 227-35.

Carrozzini Giovanni, “La fabbrica dei fenomeni della fisica contemporanea : sull'esperienza scientifica come ontogenesi”, in Giovanni Mari-Fabio Minazzi-Matteo Negro-Carlo Vinti (éd.), Epistemologia e soggettività. Oltre il relativismo, Florence, Firenze University Press/Centro Internazionale Insubrico, 2013, p. 161-177.

Carrozzini Giovanni, Gilbert Simondon filosofo della mentalité technique, Milan, Mimesis/Centra Internazionale Insubrico, 2011.

Duhem Ludovic, « "Entrer dans le moule”. Poḯtique et individuation chez Simondon », La Part de l'œil. Formes et forces. Topologies de l'individuation, Deleuze, Simondon, $\mathrm{n}^{\circ}$ 27-28, 2012-2013, p. 227-257.

Heidegger Martin, « Dépassement de la métaphysique », in Essais et conférences, trad. fr. André Préau, Préface de Jean Beaufret, Paris, Gallimard, 1958, p. 80-115.

Papinau David, "Naturalism", in Standford Encyclopedia of Philosophy. 
Price Huw, Naturalism without Mirrors, Oxford-New York, Oxford University Press, 2011.

Rovelli Carlo, Che cos'è il tempo ? Che cos'è lo spazio ?, Rome, Di Renzo Editore, 2004.

Rovelli Carlo, La realtà non è come ci appare. La struttura elementare delle cose, Milan, Raffaello Cortina Editore, 2014.

Rovelli Carlo, “Non possiamo non dirci naturalisti”, Il Sole 24 Ore, 14 décembre 2014, n 343, p. 27.

Rovelli Carlo, Sette brevi lezioni di fisica, Milan, Adelphi, 2014.

Simondon Gilbert, « Fondements de la psychologie contemporaine », in Sur la psychologie, Paris, Puf, 2015, p. 19-270.

Simondon Gilbert, « Humanisme culturel, humanisme négatif, humanisme nouveau », in Association Guillaume Budé, Congrès de Tours et Poitiers. 3-9 septembre 1953. Actes du Congrès, Paris, Les Belles Lettres, 1954, p. 51-54.

Simondon Gilbert, L'individuation à la lumière des notions de forme et d'information, Préface de Jacques Garelli, Grenoble, Éditions Jérôme Millon, 2005.

\section{NOTES}

1. David Papinau, "Naturalism", in Standford Encyclopedia of Philosophy, p. 1 (je traduis). http://plato.standford.edu/entries/naturalism/

2. Id.

3. Huw Price, Naturalism without Mirrors, Oxford-New York, Oxford University Press, 2011, p. 9 (je traduis). Dans cet ouvrage, Price définit aussi sa perspective philosophique dans le cadre du naturalisme comme un expressivisme global.

4. Ibid., p. 5 (je traduis).

5. Ibid., p. 32 (je traduis).

6. Carlo Rovelli, "Non possiamo non dirci naturalisti”, Il Sole 24 Ore, 14 décembre 2014, n $^{\circ} 343$, p. 27 (je traduis).

7. Carlo Rovelli, Sette brevi lezioni di fisica, Milan, Adelphi, 2014, p. 83-85 (je traduis).

8. Gilbert Simondon, «Humanisme culturel, humanisme négatif, humanisme nouveau ", dans Association Guillaume Budé, Congrès de Tours et Poitiers. 3-9 septembre 1953. Actes du Congrès, Paris, Les Belles Lettres, 1954, p. 52.

9. Il ne faut pas confondre cette opposition de Simondon à l'anthropologie philosophique avec un refus de l'anthropologie en tant que science humaine. Simondon juge celle-ci extrêmement importante dans le cadre de ce qu'il nomme l'« axiomatisation» - non formelle - des sciences humaines, comme le montrent la proximité de ses travaux avec les recherches anthropologiques menées par Leroi-Gourhan, ainsi que son appréciation sur la contribution de Leenhardt et les idées de Georges Gusdorf à l'égard de l'anthropologie, exprimées dans "Métaphysique et anthropologie" (Revue de Métaphysique et de Morale, 52 ${ }^{\mathrm{e}}$ Année, $\mathrm{n}^{\circ}$ 3-4, juillet-octobre 1947, p. 259-276); voir Simondon, «Fondements de la psychologie contemporaine», dans Sur la psychologie, Paris, P.U.F., 2015.

En ce qui concerne plus spécifiquement les rapports entre psychologie et anthropologie en vue d'une « axiomatisation » des sciences humaines, Simondon écrit : «Leroi-Gourhan et Leenhardt apportent une connaissance de l'homme qui n'est pas étrangère à la psychologie ; la psychologie est une science anthropologique au sens large, comme l'affirmait Gusdorf il y a dix ans » (Ibid., p. 249).

10. Carlo Rovelli, « Non possiamo non dirci naturalisti », op. cit. (je souligne et je traduis). 
11. Si l'on souligne ici le caractère masqué ou caché de l'anthropologie philosophique chez Heidegger, c'est parce que dans des notes de travail écrites entre 1936 et 1946, Heidegger avait affirmé à ce sujet: «[la] philosophie qui a cours à l'époque de la métaphysique achevée est l'anthropologie. [...] Devenue anthropologie, la philosophie elle-même périt du fait de la métaphysique " (Martin Heidegger, "Dépassement de la métaphysique ", dans Essais et conférences, trad. fr. André Préau, Préface de Jean Beaufret, Paris, Gallimard, 1958, p. 99-100. C'est la raison pour laquelle, comme on le remarquera bientôt, Anders l'accuse d'avoir participé à l'anthropologie philosophique sans en avoir conscience.

12. Günther Anders, Die Kirschenschlacht. Dialoge mit Hannah Arendt, München, Verlag C.H. Beck oHG, 2012, p. 30-32 (souligné par l'auteur ; je traduis).

13. Ibid., p. 9 (souligné par l'auteur).

14. Jean-Hugues Barthélémy a publié une lecture très attentive de la question de la «nonanthropologie » chez Simondon et Heidegger, non-anthropologie qui constitue un terrain commun aux deux penseurs sur le thème de la technique. Mais il a dans le même temps souligné à juste titre leurs divergences à propos de l'anthropologie philosophique telle qu'elle concerne, non plus la technique, mais l'homme. Voir Jean-Hugues Barthélémy, «La question de la nonanthropologie ", dans Jean-Marie Vaysse (éd.), Technique, monde, individuation. Heidegger, Simondon, Deleuze, Georg Olms Verlag, 2006.

15. Gilbert Simondon, «Fondements de la psychologie contemporaine », op. cit., p. 77.

16. Gaston Bachelard, La philosophie du non. Essai d'une philosophie du nouvel esprit scientifique, Paris, Puf., 1940, p. 11.

17. Gilbert Simondon, L'individuation à la lumière des notions de forme et d'information, Préface de Jacques Garelli, Grenoble, Jerôme Millon, 2005, p. 27 (souligné par l'auteur).

18. Ibid., p. 35.

19. Ibid., p. 165 (je souligne).

20. Sur ce point, voir aussi la conférence donnée en 2006 à Montréal par Alexis de Saint-Ours, "Relation and Quantum Gravity in the Light of Simondon and Bachelard's Philosophies", dont un résumé est disponible en français à l'URL suivante: http://www.spacetimesociety.org/ conferences/2006/Saint-Ours.E.doc.

21. Gilbert Simondon, L'individuation à la lumière des notions de forme et d'information, op. cit., p. 29 (souligné par l'auteur).

22. Ibid., p. 62.

23. Ibid., p. 32.

24. À ce propos, voir le très bel article de Ludovic Duhem, « "Entrer dans le moule”. Poïétique et individuation chez Simondon ", La Part de l'œil. Formes et forces. Topologies de l'individuation, Deleuze, Simondon, $\mathrm{n}^{\circ}$ 27-28, 2012-2013, p. 227-257.

25. Voir en particulier le début du troisième chapitre de l'ouvrage de Jean-Hugues Barthélémy, Penser l'individuation. Simondon et la philosophie de la nature, Paris, L'Harmattan, 2005, p. 99-103. Voir également le premier chapitre de son Simondon ou l'encyclopédisme génétique, Paris, Puf., 2008, p. 9-34. Sur Simondon en général, voir J.-H. Barthélémy, Simondon, Paris, Les Belles Lettres, 2014.

26. Voir Vincent Bontems, Bachelard, Paris, Les Belles Lettres, 2012.

27. Gilbert Simondon, «Fondements de la psychologie contemporaine », op. cit., p. 91.

28. Gilbert Simondon, L'individuation à la lumière des notions de forme et d'information, op. cit., p. 27.

29. Ibid., p. 62.

30. Ibid., p. 63 (souligné par l'auteur).

31. Ibid., p. 134.

32. Carlo Rovelli, Che cos'è il tempo? Che cos'è lo spazio?, Rome, Di Renzo Editore, 2004, p. 19-20 (je traduis).

33. Carlo Rovelli, La realtà non è come ci appare. La struttura elementare delle cose, Milan, Raffaello Cortina Editore, 2014, p. 118-119 (je traduis). 
34. Ibid., p. 121 (je traduis).

35. Ibid., p. 152.

36. Ibid., p. 167.

37. Carlo Rovelli, Che cos'è lo spazio ? Che cos'è il tempo, op. cit., p. 61.

38. Gilbert Simondon, « Humanisme culturel, humanisme négatif, humanisme nouveau », op. cit., p. 54 .

\section{RÉSUMÉS}

Dans cet article, nous examinerons la contribution de Gilbert Simondon au naturalisme. La première partie discutera les implications non anthropocentriques et non anthropologiques du naturalisme simondonien à l'horizon du naturalisme ontologique subjectif tel que défini par Huw Price. La deuxième partie expliquera en quoi la notion de relation est complètement repensée par Simondon. La troisième partie établira une proximité entre la pensée de Simondon et de récentes hypothèses émises par des physiciens sur la gravité quantique à partir de la notion de « relation constituante ».

INDEX

Mots-clés : anthropologie philosophique, anthropocentrisme, humanisme, naturalisme subjectif, théorie de la gravité quantique, théorie des boucles, relation constituante

\section{AUTEUR}

\section{GIOVANNI CARROZZINI}

Giovanni Carrozzini est docteur en histoire de la philosophie de l'université du Salento (Lecce), membre du CIDES (Centre International des Études Simondoniennes) et chercheur associé au Centro Internazionale Insubrico Giulio Preti e Carlo Cattaneo, université de l'Insubria (Varèse). Il a traduit des articles de Simondon, auquel il a consacré une quinzaine d'articles et il est aussi l'auteur de Gilbert Simondon filosofo della mentalité technique (Milan, 2011) et Gilbert Simondon : per un'assiomatica dei saperi. Dall' "ontologia dell'individuo" alla filosofia della tecnologia (Manni, 2006). Il a édité le numéro spécial de la revue Il Protagora consacré à Gilbert Simondon filosofo delle tecniche (XXXVI, luglio-dicembre 2008, quinta serie, $\mathrm{n}^{\circ} 12$ ), giovannicarrozzini@gmail.com. 